\title{
Diagnosis in a snap: a pilot study using Snapchat in radiologic didactics
}

\author{
Bradley Spieler $^{1}$ (I) $\cdot$ Catherine Batte $^{2} \cdot$ Dane Mackey $^{1} \cdot$ Caitlin Henry $^{1} \cdot$ Raman Danrad $^{1} \cdot$ Carl Sabottke $^{1} \cdot$ \\ Claude Pirtle $^{1}$ - Jason Mussell ${ }^{3}$. Eric Wallace ${ }^{1}$
}

Received: 9 May 2020 / Accepted: 20 July 2020 / Published online: 30 July 2020

(C) American Society of Emergency Radiology 2020

\begin{abstract}
Purpose To evaluate Snapchat, an image-based social media platform, as a tool for emergency radiologic didactics comparing image interpretation on mobile devices with conventional analysis on a classroom screen.

Materials and methods Seven radiology residents (4 juniors, 3 seniors; 4 males, 3 females; 28.4 years old, \pm 1.7 years) were shown 5 emergent radiologic cases using Snapchat and 5 cases of similar content and duration on a classroom projector over 4 weeks. All images depicted diagnoses requiring immediate communication to ordering physicians. Performance was scored 0 $2(0=$ complete miss, $1=$ major finding, but missed the diagnosis, $2=$ correct diagnosis $)$ by two attending radiologists in consensus.

Results All residents performed better on Snapchat each week. In weeks 1-4, juniors scored 21/40 (52.5\%), 23/40 (57.5\%), 19/ $40(47.5 \%)$, and 18/40 (45\%) points using Snapchat compared with 13/40 (32.5\%), 23/40 (57.5\%), 14/40 (35\%), and 13/40 $(32.5 \%)$, respectively, each week by projector, while seniors scored 19/30 (63.3\%), 21/30 (70\%), 27/30 (90\%), and 21/30 (70\%) on Snapchat versus 16/30 (53.3\%), 19/30 (63.3\%), 20/30 (66.7\%), and 20/30 (66.7\%) on projector. Four-week totals showed juniors scoring $81 / 160(50.6 \%)$ on Snapchat and 63/160 (39.4\%) by projector compared with seniors scoring $88 / 120(73.3 \%)$ and $75 / 120(62.5 \%)$, respectively. Performance on Snapchat was statistically, significantly better than via projector during weeks 1 and 3 ( $p$ values 0.0019 and 0.0031$)$.

Conclusion Radiology residents interpreting emergency cases via Snapchat showed higher accuracy compared with using a traditional classroom screen. This pilot study suggests that Snapchat may have a role in the digital radiologic classroom's evolution.
\end{abstract}

Keywords Resident education · Social media · Information technology $\cdot$ Emergency radiology

\author{
Bradley Spieler \\ bspie1@1suhsc.edu \\ Catherine Batte \\ cbatte3@gmail.com \\ Dane Mackey \\ danemackey@gmail.com \\ Caitlin Henry \\ chenry0724@gmail.com \\ Raman Danrad \\ rdanra@1suhsc.edu \\ Carl Sabottke \\ cfs121090@gmail.com
}

Claude Pirtle

cpirtl@gmail.com

Jason Mussell

jmusse@1suhsc.edu

Eric Wallace

ewalla@1suhsc.edu

1 Diagnostic Radiology, Louisiana State University Health Sciences Center, 1542 Tulane Avenue, Room 343, New Orleans, LA 70112, USA

2 Department of Physics \& Astronomy, Louisiana State University, 459-B Nicholson Hall, Tower Drive, Baton Rouge, LA 70803, USA

3 Cell Biology and Anatomy, Louisiana State University Health Sciences Center, 1901 Perdido Street, New Orleans, LA 70118, USA 


\section{Introduction}

Adaptation to altered curricular landscapes through innovative teaching methods and connectivity with students is critical in education. This is underscored by new challenges to educators that the COVID-19 pandemic has presented [1, 2]. Interaction is crucial and is the basis of all social media platforms. Social media is becoming a well-established educational tool in the field of medicine [3-7]. It is only natural that the technologically bound craft of radiology continues to push this medium in innovative ways beyond what is currently known. Collaborative efforts and learning at a distance have gained increased attention in the radiologic community [8-10]. Throughout the hospital, the usage of mobile devices for the betterment of healthcare is ubiquitous [11-13]. Continued improvement in device technology and ready access to wireless Internet can allow for constant access to high-resolution radiologic images for educational purposes for radiology trainees [14-19]. The daily usage of these items has created a high comfort level with smartphone technology and with social networking applications, facilitating its integration into graduate medical education $[6,20,21]$.

Shaping and sharing content fluidly in a specialty like radiology, which communicates with others through imaging, are reflexive. It is therefore fitting that social media has a part in the evolution of radiology, specifically as it pertains to the academic community. The integration of social media into academic radiology continues to develop and has gained much traction as a dynamic tool for medical education in recent years [7, 22]. To date, most of this discussion has primarily revolved around Twitter, Facebook, YouTube, and Instagram [6, 7, 10, 22-25]. Nonetheless, new and exciting applications for social media in medical education and in particular radiology have continued to advance beyond the confines of these social media staples [18].

Snapchat, a camera-based application that allows social media networking via video and digital images with text messaging functionality, was released in 2011 and has continued to expand in usage around the globe with hundreds of millions of users worldwide. This study explores the intriguing potential of Snapchat, one of the most popular social media platforms in 2019 for young adults, as a didactic tool in radiology resident education. Our radiology resident cohort is comprised entirely of millennials, a group considered to be the most competent with smartphone technology and social media [20, 21, 26-29]. The increased utilization of smartphones and handheld devices by this demographic, specifically radiology residents, is theorized to aid in improved didactic performance and accuracy in emergency imaging diagnosis.

Our study was designed to test this hypothesis by comparing resident performance analyzing radiology cases using the Snapchat application on their smartphones compared with the more traditional model using a single screen in the resident conference room. All cases used were diagnoses considered to require emergent, non-routine communication on the order of minutes to the ordering healthcare provider. As such, these types of diagnoses demand prompt imaging recognition as they are considered critical findings which could result in mortality and significant morbidity if not acted upon expeditiously [30-33]. We chose the Snapchat platform for our investigation due to its quick acquisition of user-friendly groups, rapidity of image sharing, and for its timed image display capability. Users can select the duration that a photo will appear on screen to the viewers of their "snap" or "story." The ability to specify the length of time an image is visible to a user (or trainee in our scenario) in rendering an image-based diagnosis creates an intriguing potential for innovation in radiologic curricula, particularly as it relates to assigning a metric to diagnostic performance. To the best of our knowledge, no similar systematic empirical research involving the utility of Snapchat as a pedagogic tool in emergency radiology exists.

\section{Materials and methods}

Our institutional review board approved this study and deemed it to be exempt under 45CFR46.104(d), Category \#4.iii.. We performed a retrospective database query within the electronic medical record system at University Medical Center in New Orleans (UMCNO) for cases reflective of diagnoses warranting non-routine communication as defined by the Actionable Reporting Work Group, a body formed by the American College of Radiology to enhance and standardize the reporting of radiologic findings [30]. A variety of emergency room cases ( 40 total) felt to be typical of those requiring non-routine communication of findings were chosen in consensus by two board certified radiologists, each with greater than 5-year experience following fellowship. This sample of cases is chosen because of the rapid recognition they demand and included a mix of imaging diagnoses considered to be critical results (Tables 1, 2, 3 and 4) [30]. While we acknowledge that the selected diagnoses can be seen at any hospital, this sample was of special interest given that our institution is a high-volume level one trauma center, where members of our department are regularly consulted to "rule out" these types of diagnoses on the spot.

Over 4 weeks, a group of 7 radiology residents $((4$, junior residents defined as postgraduate year (PGY) two or three; 3 , senior residents PGY four and five) (4 males, 3 females; 28.4 years old \pm 1.7 years)) were shown 5 radiology cases for $5 \mathrm{~s}$ using Snapchat and 5 cases for $5 \mathrm{~s}$ via projector (NEC MultiSync V651 SmartBoard UF75w) each week by a blinded attending radiologist in our radiology department's classroom. A trial run with Snapchat was first performed with a test image to ensure that all participants received the "snap" 
Table $1 \quad(\mathrm{CT}=$ Computed Tomography, $\mathrm{CR}=$ Radiograph).

\begin{tabular}{|c|c|c|c|c|c|c|c|c|c|}
\hline & & iphone 8 & iphone 8 & Samsung Galasyy Note 9 & iPhone $X$ & iPhone $x$ & iPhone X & iPhone $\mathrm{X}$ & \\
\hline & CASE & JIR RES 1 & JR RES 2 & JRRES 3 & JRRES 4 & SR RES 1 & SR RES 2 & SR RES 3 & ALL \\
\hline \multirow[t]{6}{*}{ SNAPCHAT } & CT appendicitis -1 & & o & 0 & 2 & & 2 & 2 & \\
\hline & CT PE- 2 & & 0 & 2 & 2 & & 2 & 2 & \\
\hline & CXR pneumoperitoneum - 3 & & 2 & 2 & 0 & & 0 & 2 & \\
\hline & CT epidural hematoma -4 & & 1 & 1 & 2 & & 1 & 1 & \\
\hline & Xray C1-2 dislocation - 5 & & 2 & 0 & 1 & & 0 & 0 & \\
\hline & TOTAL/ 10 & & 5 & 5 & 7 & & 5 & 7 & $40 / 70(57.1 \%)$ \\
\hline \multirow{7}{*}{$\begin{array}{r}\text { PROJECTOR } \\
\text { NEC MultiSYnc V651 } \\
\text { SmartBoard UF75w }\end{array}$} & CT PV gas, mesenteric ischemia - 1 & & o] & 0 & 0 & & 0 & 0 & \\
\hline & CXR tension PTX- 2 & & 1 & 1 & 2 & & 1 & 1 & \\
\hline & CT renal laceration - 3 & & of & 0 & 1 & & 2 & 2 & \\
\hline & CT subdural hematoma -4 & & 2 & 1 & 2 & & 2 & 2 & 12 \\
\hline & CT visceral perforation -5 & & of & 1 & 0 & & 0 & 0 & \\
\hline & TOTAL/10 & & 3 & 3 & 5 & & 5 & 5 & $29 / 70(41.4 \%)$ \\
\hline & TOTAL/ 20 & & 8 & 8 & 12 & & 10 & 12 & $69 / 140(49.3 \%)$ \\
\hline
\end{tabular}

Table 2 (CT = Computed Tomography, $\mathrm{CR}=$ Radiograph, US = Ultrasound).

\begin{tabular}{|c|c|c|c|c|c|c|c|c|c|c|}
\hline & & CASE & $J R \operatorname{Res} 1$ & JR Res 2 & $J R \operatorname{Res} 3$ & $J R$ Res 4 & SR Res 1 & SR Res 2 & SR Res 3 & \\
\hline \multirow{12}{*}{$\overline{\text { WEEK } 2}$} & \multirow[t]{6}{*}{ SNAPCHAT } & CXR rib fractures, child abuse -1 & 1 & 1 & 0 & 1 & 1 & 1 & 0 & \\
\hline & & CT subarachnoid hemorrhage, skull frx - 2 & 7 & 1 & 2 & 2 & 2 & 1 & 2 & \\
\hline & & Xray cecal volvulus -3 & 1 & 2 & 2 & 0 & 2 & 2 & 2 & \\
\hline & & US ovarian torsion - 4 & 2 & 0 & 2 & 0 & 0 & 2 & 2 & \\
\hline & & CT Fournier's gangrene -5 & 2 & 0 & 2 & 0 & 0 & 2 & 2 & \\
\hline & & TOTAL $/ 10$ & 8 & 4 & 8 & 3 & 5 & 8 & 8 & $44 / 70(62.9 \%)$ \\
\hline & \multirow[t]{7}{*}{$\begin{array}{ll}\text { PROJECTOR } \\
\end{array}$} & CT Intraperitoneal bladder rupture - 1 & 1 & 0 & 1 & 2 & 1 & 0 & 0 & \\
\hline & & Xray metaph corner ffx, child abuse - 2 & 1 & 0 & 0 & 2 & 2 & 1 & 2 & \\
\hline & & Xray SBO -3 & 2 & 2 & 2 & 2 & 2 & 2 & 2 & \\
\hline & & CT pneumocephalus - 4 & 4 & 0 & 2 & 0 & 2 & 2 & 2 & \\
\hline & & CT closed loop obstruction - 5 & 1 & 0 & 1 & 2 & 0 & 0 & 1 & \\
\hline & & TOTAL/10 & 7 & 2 & 6 & 8 & 7 & 5 & 7 & $42 / 70(60.0 \%)$ \\
\hline & & TOTAL/20 & 15 & 6 & 14 & 11 & 12 & 13 & 15 & $86 / 140(61.4$ \\
\hline
\end{tabular}

Table 3 (CT = Computed Tomography, $\mathrm{CR}=$ Radiograph, US = Ultrasound $)$.

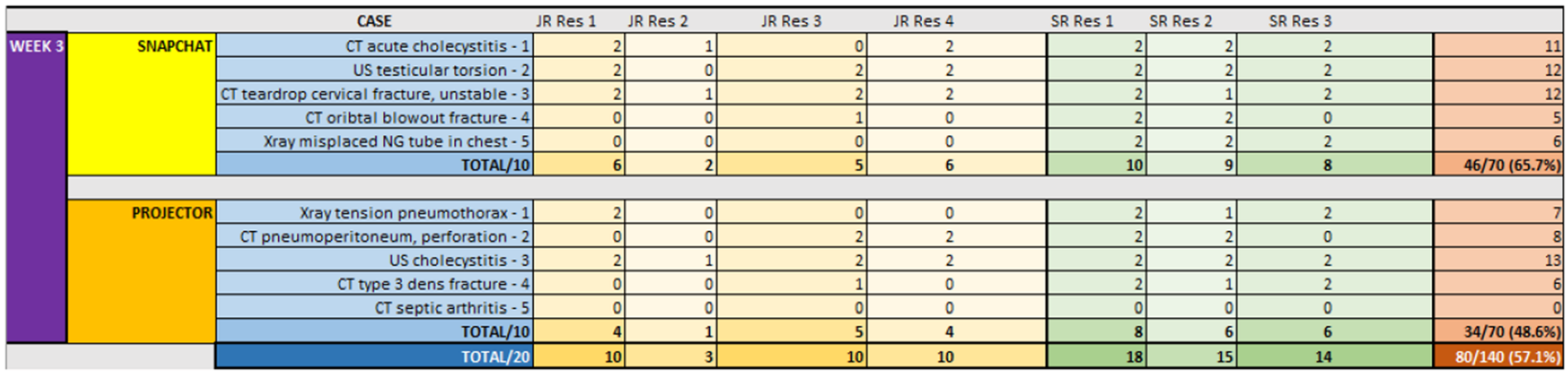

Table 4 (CT = Computed Tomography, $\mathrm{CR}=$ Radiograph, US = Ultrasound, Fluoro $=$ Fluoroscopy $).$

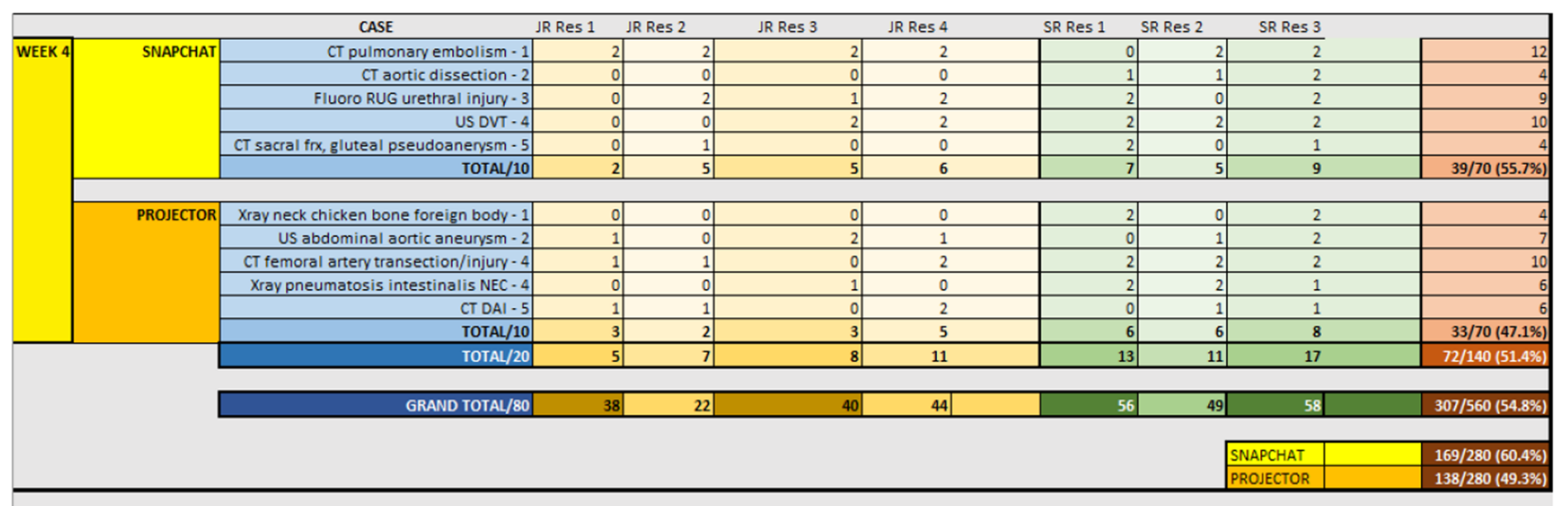


simultaneously and understood the process. Of note, all participants expressed familiarity with Snapchat prior to the trial run. Images were shown sequentially via projector immediately following the Snapchat phase of exhibition. A 5-s duration of visibility was preselected in the Snapchat app prior to sending or "snapping" the image. As such, images would disappear from their screen after the $5 \mathrm{~s}$. Similarly, during the projector phase of exhibition, after $5 \mathrm{~s}$ the slide was advanced to a blank screen before displaying the next case. All images used depicted situations in which immediate or urgent communication of the findings to the ordering physician was necessary in accordance with best practice guidelines [30]. No history was given prior to exhibition of the images. For each case shown on Snapchat, a companion case illustrating a similar imaging finding was shown via the conventional method on the projector to all subjects over the course of 4 weeks. Subjects recorded their diagnosis for each case via free text on a data sheet that was collected at the end of each session. Furthermore, the cases were randomized such that a complimentary case shown in week 1 , for example, may be shown at any of the 4-week intervals to mitigate familiarity bias. For instance, an example of intracranial hemorrhage as depicted by a subdural hematoma (Fig. 1) is exhibited on the classroom

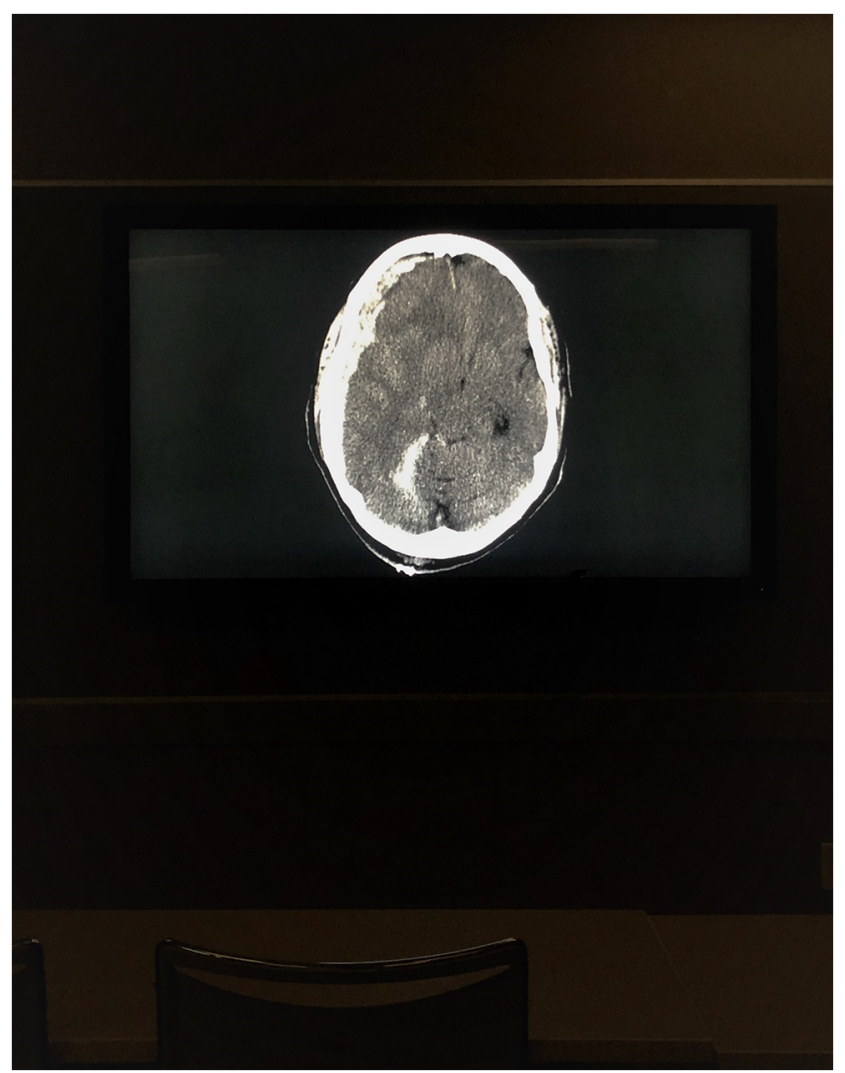

Fig. 1 Photograph demonstrating the point of view of resident viewing a case on the classroom projector, a non-contrast $\mathrm{CT}$ of the head showing a right-sided crescent-shaped high-density extra-axial collection compatible with a subdural hematoma. A tentorial subdural hematoma is also seen. There is associated mass effect and right to left midline shift projector in week 1 , and a case of a subarachnoid hemorrhage (Figs. 2 and 3) is shown via Snapchat in week 2. Resident performance was scored $0-2(0=$ complete miss; $1=$ had major finding, but missed the diagnosis; $2=$ correct diagnosis) by the supervising radiologists in consensus. For example, with a case of a pneumothorax resulting in marked mediastinal shift (Fig. 4), the subject was granted one point if they answered only "pneumothorax" but two if they answered "tension pneumothorax." Statistical analysis was performed using Microsoft Excel software (Microsoft Corporation. [2018]. Microsoft Excel). A two-tailed paired $t$ test was used to analyze resident performance on Snapchat vs projector. Supplemental statistical analysis was also performed in Python 3 (https://www.python.org/). For pairing purposes during statistical analysis, pairs were formed based on individual residents and the time period of evaluation (weeks 1-4). Cases were pooled by week for pairing consideration as cases were similar in complexity but not identical. When appropriate due to sufficient sample size (greater than 20 samples available), Mann-Whitney $U$ test and Wilcoxon signed-rank test were performed to supplement the paired $t$ test. D'Agostino-Pearson test was also performed to assess for normality in the data distributions.

\section{Results}

Both junior and senior residents performed better on Snapchat each week when compared with the projector. At week 1, junior residents scored 21 out of $40(52.5 \%)$ possible points using Snapchat compared with 13 out of $40(32.5 \%)$ via projector; while the senior residents scored 19/30 (63.3\%) on Snapchat versus 16/30 (53.3\%) using the projector. At week 2 , junior residents scored 23/40 (57.5\%) evaluating both Snapchat and projector images, and senior residents scored 21/30 (70\%) interpreting Snapchat images versus 19/30 (63.3\%) on the projector. At week 3, junior residents scored $19 / 40(47.5 \%)$ reading Snapchat images and 14/40 (35\%) reading projector images, and senior residents scored 27/30 (90\%) on Snapchat and 20/30 (66.7\%) via the projector. At week 4, junior residents scored 18/40 (45\%) on Snapchat and $13 / 40(32.5 \%)$ on projector images, and senior residents scored 21/30 (70\%) via Snapchat and 20/30 (66.7\%) reading projector images. The 4-week totals show that junior residents scored 81/160 (50.6\%) reading Snapchat images and only 63/ $160(39.4 \%)$ reading projector images, and the senior residents scored a total of 88/120 (73.3\%) on Snapchat compared with $75 / 120(62.5 \%)$ reading projector images. During weeks 1 and 3 , the residents' performance using Snapchat for image interpretation was statistically, significantly better than performance via projector with $p$ values of 0.0019 and 0.0031 , respectively (used a two-tailed paired $t$ test). Two junior residents (JR Res 2 and JR Res 3) and one senior resident (SR Res 
Fig. 2 Screen capture of the Snapchat application from the point of view of the attending radiologist, a non-contrast $\mathrm{CT}$ of the head showing hyperdense material compatible with blood filling the suprasellar cistern with peripheral extension, consistent with subarachnoid hemorrhage (a). b shows a screenshot of the Snapchat app following clicking of "send photo" from the attending's point of view, immediately before the image is sent to the radiology resident group

Fig. 3 Screen capture of the same image depicted in Fig. 2 from the resident's point of view when receiving the image via Snapchat (a). $\mathbf{b}$ is a photograph of a resident's phone when viewing the image via snap chat

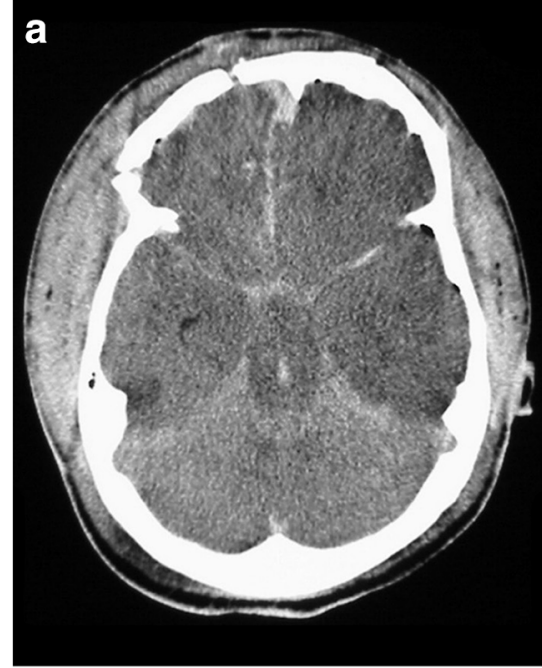

b< Q \& Radiology Resid... \& GROUPS SELECTED

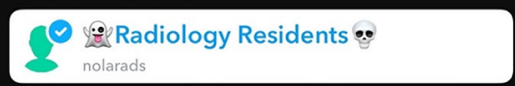

^ Export Photo

每 Delete Photo

(3) My Eyes Only

$0^{\circ}$ Edit Photo

Send Photo

U: Radiology Residents

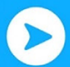




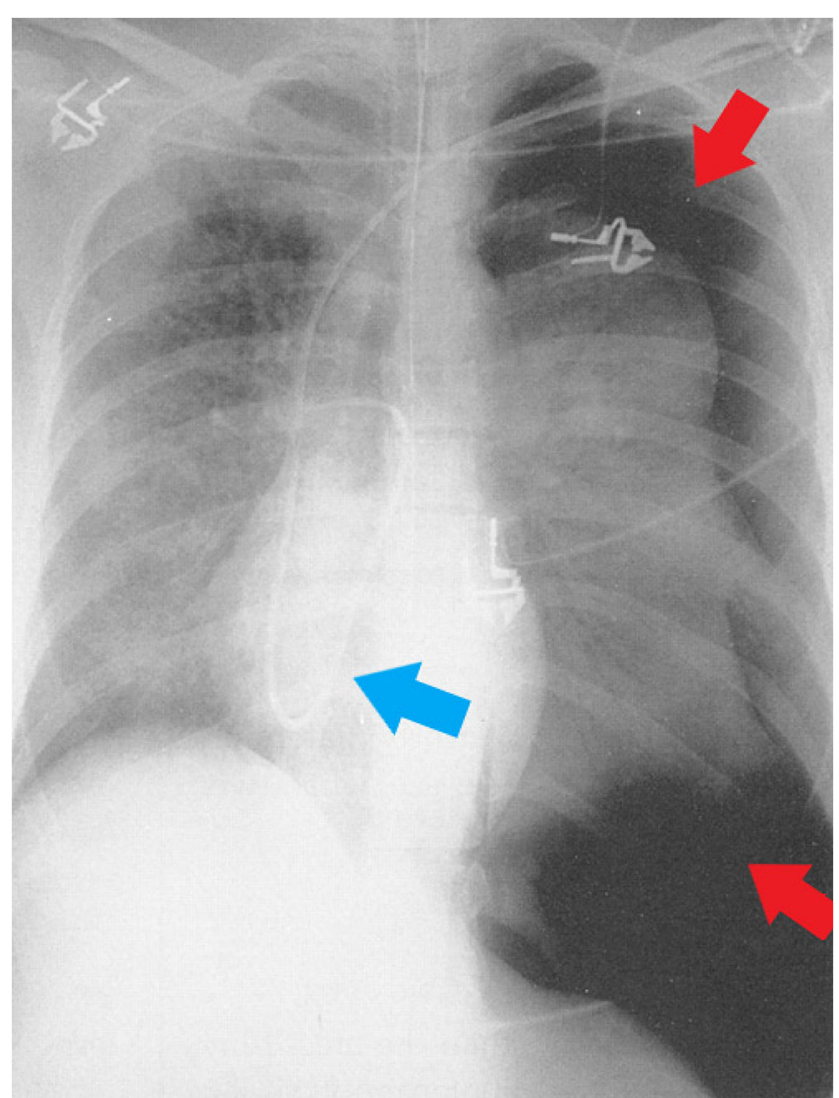

Fig. 4 Anteroposterior radiograph of the chest showing a large left-sided pneumothorax (red arrows) resulting in compression of the left lung and mediastinal shift to the right, including that of the heart and a Swan-Ganz catheter (blue arrow)

3) performed better on Snapchat than projector all 4 weeks, and these results were statistically significant with $p$ values of $0.008,0.029$, and 0.007 , respectively, using a one tail $t$ test and $p$ values of $0.016,0.058$, and 0.014 , respectively, using a two-tailed paired $t$ test. The results for the three residents with statistically different performance on Snapchat versus

Fig. 5 Two junior residents (JR Res 2 and JR Res 3 ) and one senior resident (SR Res 3) performed better on Snapchat than projector all 4 weeks, and these results were statistically significant with $p$ values of 0.008 , 0.029 , and 0.007 , respectively, using a one tail $t$ test and $p$ values of $0.016,0.058$, and 0.014 , respectively, using a two-tailed paired $t$ test. The results for the three residents with statistically different performance on Snapchat versus the projector projector are shown (Fig. 5), and the resident results that were not statistically significant are shown (Fig. 6). All resident data for all 4 weeks is shown on Tables 1, 2, 3 and 4.

Combined scores on projector and Snapchat presentation across all weeks and residents (Table 4) are 160/280 (57\%) for Snapchat compared with 130/280 (46\%) for projector-based presentation. Cases were pooled by week and by resident. Two-tailed paired $t$ testing was significant for superiority of Snapchat performance with $p$ value of 0.002 . Pairing was considered appropriate due to similar case complexity, but Mann-Whitney $U$ testing was also performed with $p$ value of 0.036 , thus also favoring statistically significant superior performance with Snapchat. Notably, though, Wilcoxon signedrank test, performed as a non-parametric variant of the paired $t$ test, did not show statistical significance ( $p$ value of 0.075 ), likely due to our small sample size. Nevertheless, D'AgostinoPearson testing for normality did not show any significantly non-normal distribution features in either our Snapchat data (D'Agostino-Pearson $\mathrm{p}$ value of 0.86 ) or projector data (D'Agostino-Pearson $\mathrm{p}$ value of 0.44 ) which would indicate inappropriateness of performing a paired $t$ test compared with the Wilcoxon signed-rank test [34-36].

\section{Discussion}

While there is no clear correlation between the speed of radiologic interpretation and overall accuracy in the literature, knowledge of the speed at which a novice recognizes key imaging findings could provide insight into understanding their search pattern and ultimately aid in its refinement [37]. All residents faced with emergent radiologic diagnoses at the same set time interval via Snapchat method consistently demonstrated higher accuracy across the 4-week testing period compared with similar difficulty cases displayed on a

\section{Weekly Individual Performance Snapchat vs Projector STATISTICALLY SIGNIFICANT}

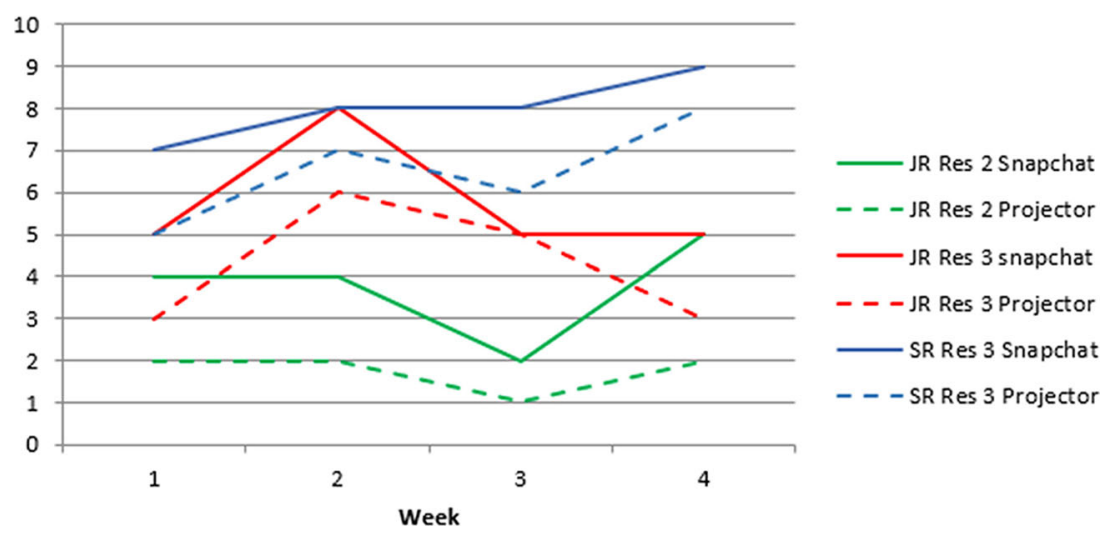


Fig. 6 The results for the four residents without statistically different performance on Snapchat versus the projector
Weekly Individual Performance Snapchat vs Projector NOT STATISTICALLY SIGNIFICANT

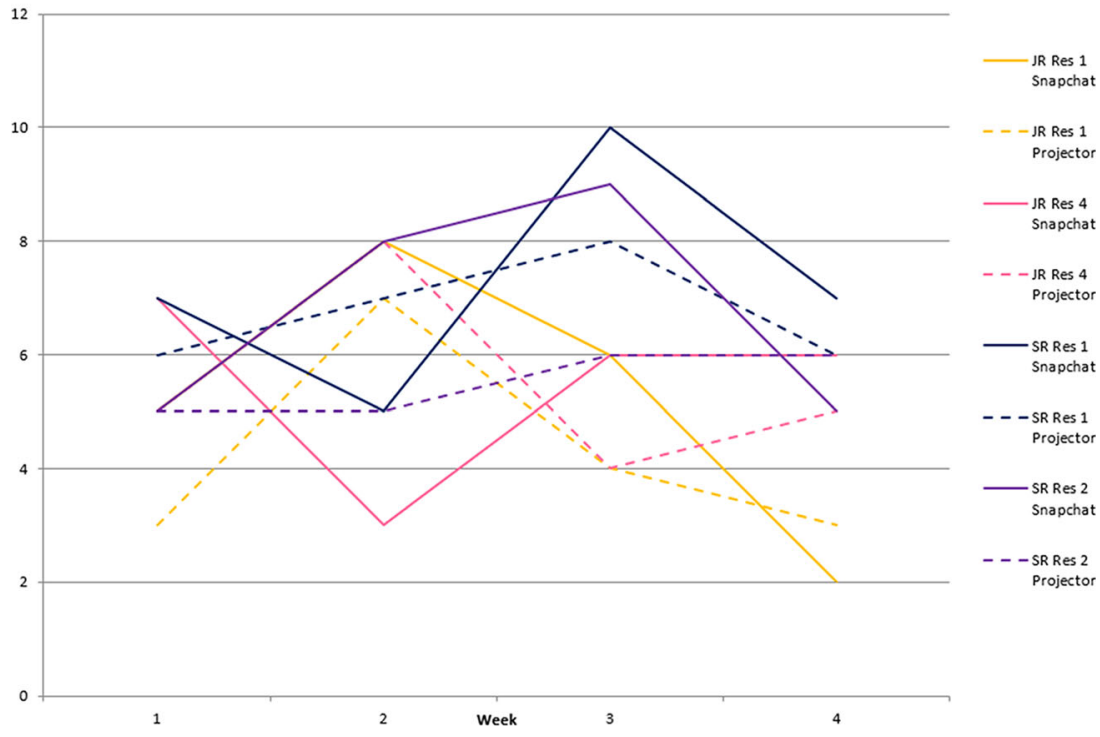

traditional classroom projector screen (Fig. 7). As per statistical analysis, these findings were significant in the junior group but not in the senior group. This discrepancy is most likely related to a small sample size (3 seniors). But questions regarding age of participants and their associated likelihood of social media smartphone usage are raised. Of note, two of the senior residents were over the age of 30 [38]. This is notable as over $75 \%$ of Snapchat users under the age of 29 have been reported to use the app daily [29] with recent data suggesting that the percentage of users in the USA declines from 62 to $25 \%$ after the age of 29 [38]. There are potentially many uncontrolled variables in our study design; the findings of this study raise attention-grabbing inquiries regarding imaging interpretation on personal devices, particularly of the handheld variety. The potential differences in one's perception of a 4.7 to 6.4-in. screen held within arm's length versus that of a 65 - in. screen viewed at approximately 10 to $15 \mathrm{ft}$ are intriguing. Advances in smartphone technology, specifically recent improvements in small screen resolution, raise interesting questions regarding the abilities of human visual recognition and the learning process. Many smartphones keep track of how long certain applications are on screen per a 24-h or 7-day period, alerting users as to which applications they use most. Nearly a quarter of adults in the USA report using Snapchat on their mobile devices, and of those, the majority is millennials who are under 30 years of age. "Active" users of the app access it roughly 30 times daily $[29,38]$. The high-definition display offered by modern devices provides detailed visual content, namely, photographs, videos, and figures. Constant usage of one's personal device and familiarity with imagebased apps such as Snapchat compared with the much lower visual attention devoted to the classroom projector screen in
Fig. 7 All residents faced with critical imaging diagnoses via Snapchat consistently demonstrated higher accuracy across the 4-week testing period compared with cases displayed on a traditional classroom projector screen. During week 1 and week 3 , the residents' performance using Snapchat for diagnoses was statistically significantly better than performance via projector with $p$ values of 0.0019 and 0.0031 , respectively (used a twotailed paired $t$ test)
Total Score by Week

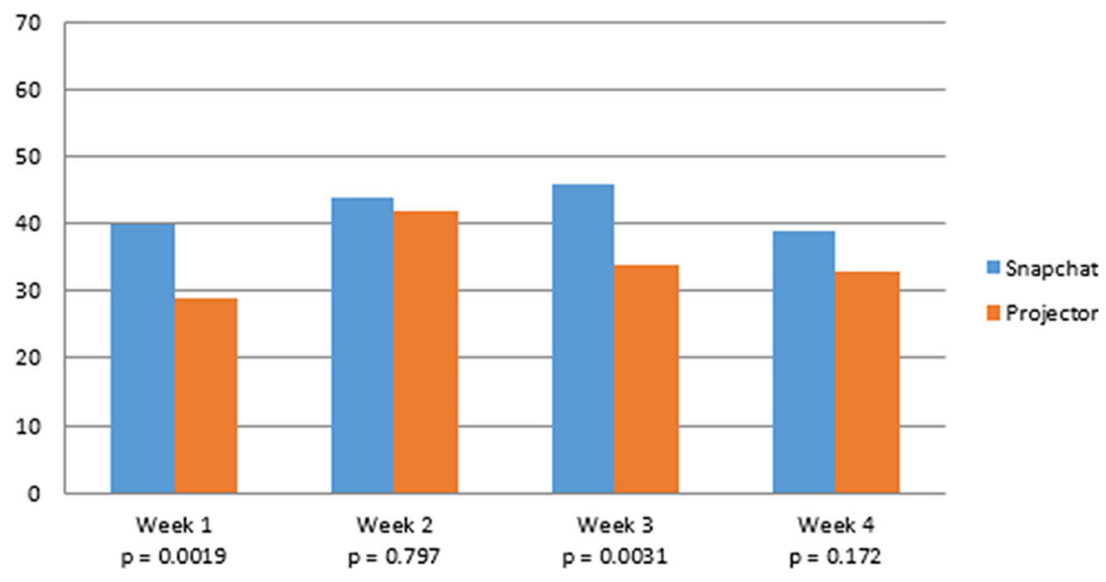


each subject's daily life may explain the relative increased diagnostic accuracy seen in the resident Snapchat sessions. It is our speculation that it may be facile to interpret images on a device that one is more familiar with in the context of software that is estimated to be accessed readily by young millennials, as in this study (this cohort),over 12 times a day $[29,38]$.

Recent literature has highlighted a need for timely and efficient mechanisms for educating radiologists and disseminating rapidly evolving clinical information in attempts to ensure accurate diagnoses and increase resident autonomy in the changing landscape of graduate medical education [39-42].

A recent review summarized the importance of visual recognition of abnormalities as a more accurate metric of achievement of expert status in radiologists over gaze tracking, based on the variable patterns seen in radiologists [42].The Snapchat platform provides the unique possibility to design a series of modules to expose learners to a series of normal cases, accompanied by an abnormal case of an infinite variety of diseases. These time-limited exposures followed by concise explanations from attendings identifying the abnormality and its diagnostic features could be used as immediate feedback for learners. Frequency of success in learners could allow senior trainers to assess when learners are ready to progress to subsequent modules and for residents, possibly when they can be given more autonomy within their training programs (39, Waite). Further studies are needed to test this hypothesis. With regard to rapidly disseminating new clinical information, Bai et al. compared the ability of radiologists to differentiate COVID-19 from other viral pneumonias across 58 age-matched cases [43]. The accuracy of the radiologists in this study ranged from 53 to $97 \%$, and the radiologist experience levels ranged from 5 to 25 years in practice. Notably, the second most experienced radiologist (20 years) was the only participant who did not complete some form of COVID-19-specific training prior to the study, and this radiologist had the lowest accuracy at 53\% compared with the average accuracy of $87 \%$ for the 6 other participants. This work reinforces the importance of targeted education to improve radiologist performance. Understanding ways to improve the effectiveness of such disease-targeted training within a radiology department is critical, and here, we are able to demonstrate the potential value of a tool like Snapchat for tailoring the delivery method of training cases based on a learner's potential age or level of training-based preferences.

Our pilot training exercise could be used to maintain engagement with trainees outside of the classroom. Residents at our institution, for example, often rotate at different hospitals, rendering attendance of daily lectures at our main site difficult. Snapchat provides an opportunity to maintain active engagement in teaching exercises with trainees that are physically distant beyond teleconferencing. Also, this method raises intriguing possibilities for teaching outside of the classroom in the form of testing such as "pop quizzes." Snapchat's user- friendly format, accessibility on mobile devices, and familiarity with the age cohort of the typical radiology resident population provide for a potentially useful tool in resident education, such as in overnight coverage scenarios. Snapchat may have adaptability to function as a platform for quick review of cases in an effort to counteract the perceived negative impact on training by 24-h attending coverage. Snapchat could be used to quickly review cases that might not have otherwise been seen by a resident on call in tandem with an attending, particularly if they are in a different location. This technique would be similar to what the Medical College of Wisconsin's Department of Radiology has employed with teleconferencing software overnight [41]. Furthermore, the ability to set a strict duration of image visibility on Snapchat can also be a strength for testing purposes in that students will have little to no available time for consultation, particularly if responses are requested shortly after image visibility. Moreover, this could allow for gauging improvement over time, in particular with respect to the speed of a given pattern recognition [44] This is in contrast to the testing of unknown cases via other imagebased social media platforms such as Twitter and Instagram in which the duration of an image's visibility cannot be timed. Also with these applications, all users accessing the image can freely text in the comment section, potentially biasing a test taker [23]. With Snapchat, a group of users can be sent an image simultaneously, and the host can be set as the only one who can see comments.

Our study had the following limitations. First, this was a pilot investigation with a small sample size and narrow age range of participants. Also, while each subject's personal device was handheld and equipped with similar screen size, they were not all the exact same. All however were new-generation iPhone models except for one subject that used a newgeneration Samsung Galaxy. The phones were slightly different in size with the Samsung device having a screen size of $6.4 \mathrm{in}$. and the iPhones having a screen size ranging from 4.7 to 5.85 in. $[45,46]$. Other factors such as differences in screen resolutions and brightness of each of the individual screens such as the back-lit screen display settings were not accounted for in our study. However, it was felt by the faculty that the luminescence of all of the devices was adequate for viewing radiological imaging [45], [46, 47]. The same screen and room lighting were used for the projector phase of each session. Additionally, while efforts were made to nullify familiarity biases, it is possible that subjects may have learned certain imaging patterns and applied them on a later case, thus allowing them to score higher on the subsequent companion case. For example, the visualization of a radiograph of a traumatic subluxation at C1-2 shown on Snapchat in week 1 could have aided a subject in week 3 when confronted with a $\mathrm{CT}$ of a dens fracture on the projector. Also, given that our case collection was comprised of both cross-sectional as well as non-cross-sectional modalities, only static images were 
displayed to be consistent in our methodology. We recognize that the use of video could simulate scrolling and possibly make for a more realistic experience. A concern to this approach, however, would be that differences in individual rates of scrolling could limit the study. This might be mitigated by accessing the DICOM files but would then be inaccessible via the Snapchat app to our knowledge. Finally, some of residents may have had more acquaintance with certain imaging diagnoses through rotation schedules and study patterns than others despite being at a similar academic rank, enabling them to be more skillful at imaging interpretation regardless of the screen that images were viewed upon.

Results from this pilot study could facilitate a promising and novel radiologic training method in enhancing recognition of imaging diagnoses, particularly those of lifethreatening nature, which could be applied to the evolving landscape of distance learning. Moreover, the integration of handheld mobile devices could aid in bridging generational gaps in radiology departments, particularly as it relates to the growing millennial resident base. Our hope is that this investigation can aid in the promotion of active learning and lecture participation as well as to explore metrics for gauging diagnostic performance and pattern recognition in image-based curricula both within the classroom and in remote teaching formats. A larger sample size and more intricate study designs, for example, with the adjunct of eye tracking, may support the burgeoning role that handheld devices and imaged-based social media applications like Snapchat can play in learning at all levels of education especially in the context emergency radiology.

Acknowledgments We are grateful for the following individuals and entities for their assistance, guidance and helpful discussions: Robin McGoey, MD, the University Medical Center New Orleans Information Technology Department, and the Louisiana Health Sciences Center Department of Radiology Residency Program.

\section{Compliance with ethical standards}

Conflict of interest The authors declare that they have no conflict of interest.

\section{References}

1. Gill SD, Stella J, Blazeska M, Bartley B (2020) Distant supervision of trainee emergency physicians undertaking a remote placement: a preliminary evaluation. Emerg Med Australas 32:446-456

2. Gewin V (2020) Five tips for moving teaching online as COVID-19 takes hold. Nature 580:295-296

3. Oyewole BK, Animasahun VJ, Chapman HJ (2020) A survey on the effectiveness of WhatsApp for teaching doctors preparing for a licensing exam. PLoS One 15(4):e0231148

4. Linzey JR, Robertson FC, Haider AS, Graffeo CS, Wang JZ, Shasby G, Alotaibi NM, Cohen-Gadol AA, Rutka JT (2020) Specialized social media team increases online impact and presence: the journal of neurosurgery experience. J Med Internet Res 22:e17741

5. Daneshjou R, Adamson AS (2020) Twitter journal clubs: medical education in the era of social media. JAMA Dermatol 156:729

6. Sterling M, Leung P, Wright D, Bishop TF (2017) The use of social media in graduate medical education: a systematic review. Acad Med 92(7):1043-1056

7. Fehring KA, De Martino I, McLawhorn AS, Sculco PK (2017) Social media: physicians-to-physicians education and communication. Curr Rev Musculoskelet Med 10(2):275-277

8. Armstrong V, Tan N, Sekhar A, Richardson ML, Kanne JP, Sai V, Chernyak V, Godwin JD, Tammisetti VS, Eberhardt SC, Henry TS (2019) Peer learning through multi-institutional web-based case conferences: perceived value (and challenges) from abdominal, cardiothoracic, and musculoskeletal radiology case conference participants. Acad Radiol

9. Richardson ML, Petscavage JM, Hunter JC, Roberts CC, Martin TP (2012) Running an online radiology teaching conference: why it's a great idea and how to do it successfully. Acad Radiol 19(6):746751

10. Chow RA, Tan N, Henry TS, Kanne JP, Sekhar A (2020) Peer learning through multi-institutional case conferences: abdominal and cardiothoracic radiology experience. Acad Radiol

11. Bhavnani SP, Narula J, Sengupta PP (2016) Mobile technology and the digitization of healthcare. Eur Heart J 37(18):1428-1438

12. Short SS, Lin AC, Merianos DJ, Burke RV, Upperman JS (2014) Smartphones, trainees, and mobile education: implications for graduate medical education. J Grad Med Educ 6(2):199-202

13. Redmond CE, Crawford R, O'Neill DC, Lee MJ (2020) Social media as a learning resource for medical students. Ir Med J 113(4):64

14. Raman B, Raman R, Raman L, Beaulieu CF (2004) Radiology on handheld devices: image display, manipulation, and PACS integration issues. Radiographics 24(1):299-310

15. Jamal A, Temsah MH, Khan SA, Al-Eyadhy A, Koppel C, Chiang MF (2016) Mobile phone use among medical residents: a crosssectional multicenter survey in Saudi Arabia. JMIR Mhealth Uhealth 4(2):e61

16. Choudhri AF, Radvany MG (2011) Initial experience with a handheld device digital imaging and communications in medicine viewer: OsiriX mobile on the iPhone. J Digit Imaging 24(2):184-189

17. John S, Poh AC, Lim TC, Chan EH, Chong le R (2012) The iPad tablet computer for mobile on-call radiology diagnosis? Auditing discrepancy in CT and MRI reporting. J Digit Imaging 25(5):628 634

18. Gupta S, Johnson EM, Peacock JG, Jiang L, McBee MP, Sneider MB, Krupinski EA (2020) Radiology, mobile devices, and internet of things (IoT). J Digit Imaging

19. European Society of R (2018) ESR paper on the proper use of mobile devices in radiology. Insights into Imaging 9(2):247-251

20. Chen P-H, Scanlon MH (2018) Teaching radiology trainees from the perspective of a millennial. Acad Radiol 25(6):794-800

21. Botterill J, Bredin M, Dun T: (2015) Millennials' media use: it is a matter of time. Can J Commun 40(3) Vicissitudes of the Cyber

22. Hawkins CM, Duszak R, Rawson JV (2014) Social media in radiology: early trends in Twitter microblogging at radiology's largest international meeting. J Am Coll Radiol 11(4):387-390

23. Ranginwala $\mathrm{S}$, Towbin AJ (2018) Use of social media in radiology education. J Am Coll Radiol 15(1 Pt B):190-200

24. Rigamonti L, Dolci A, Galetta F, Stefanelli C, Hughes M, Bartsch M, Seidelmeier I, Bonaventura K, Back DA (2019) Social media and e-learning use among European exercise science students. Health Promot Int

25. Kelly BS, Redmond CE, Nason GJ, Healy GM, Horgan NA, Heffernan EJ (2016) The use of Twitter by radiology journals: an 
analysis of Twitter activity and impact factor. J Am Coll Radiol 13(11):1391-1396

26. Clements C (2016) Snapchat's ability to build and maintain friendships. Theses, Dissertations \& Honors Papers, Paper 393

27. Ravesloot CJ, van der Schaaf MF, Kruitwagen C, van der Gijp A, Rutgers DR, Haaring C, Ten Cate O, van Schaik JPJ (2017) Predictors of knowledge and image interpretation skill development in radiology residents. Radiology 284(3):758-765

28. Snap Inc. (2019) Announces Fourth Quarter and Full Yea. Financial Results. Retrieved May 14, 2020, from [https://investor. snap.com/news-releases/2020/02-04-2020-210906650]

29. Snapchat by the Numbers: Stats, Demographics \& Fun Facts. Retrieved, May 14, 2020, from [https://www.omnicoreagency. com/snapchat-statistics/]

30. Larson PA, Berland LL, Griffith B, Kahn CE Jr, Liebscher LA (2014) Actionable findings and the role of IT support: report of the ACR actionable reporting work group. J Am Coll Radiol 11(6):552-558

31. Berlin L (2015) Communicating nonroutine radiologic findings to the ordering physician: will (should) information technologyassisted communication replace direct voice contact? Radiology 277(2):332-336

32. Berlin L (2015) MEDICOLEGAL - malpractice and ethical issues in radiology. Am J Roentgenol 204(4):W492-W492

33. Harvey HB, Alkasab TK, Pandharipande PV, Zhao J, Halpern EF, Salazar GM, Abujudeh HH, Rosenthal DI, Gazelle GS (2015) Radiologist compliance with institutional guidelines for use of nonroutine communication of diagnostic imaging results. J Am Coll Radiol 12(4):376-384

34. D'Agostino R, Pearson ES (1973) Tests for departure from normality. Empirical results for the distributions of $\mathrm{b}<\mathrm{sub}>2</ \mathrm{sub}>$ and $\sqrt{ }$ b $<$ sub $>1</$ sub $>$. Biometrika 60(3):613-622

35. Wilcoxon $F$ (1945) Individual comparisons by ranking methods. Biom Bull 1(6):80-83

36. Mann HB, Whitney DR (1947) On a test of whether one of two random variables is stochastically larger than the other. Ann Math Stat 18(1):50-60

37. Muroff LR, Berlin L (2019) Speed versus interpretation accuracy: current thoughts and literature review. AJR Am J Roentgenol 213(3):490-492
38. Social Media Fact Sheet. Retrieved May 15, 2020, from [https:// www.pewresearch.org/internet/fact-sheet/social-media/]

39. Kanne JP, Little BP, Chung JH, Elicker BM, Ketai LH (2020) Essentials for radiologists on COVID-19: an update-radiology scientific expert panel. Radiology 2005:27

40. Mossa-Basha M, Medverd J, Linnau K, Lynch JB, Wener MH, Kicska G, Staiger T, Sahani D (2020) Policies and guidelines for COVID-19 preparedness: experiences from the University of Washington. Radiology 2013:26

41. Vagvala SH, Tolat PP, Hohenwalter MD, Duvnjak P (2020) Skype as a tool for resident education on call. JAm Coll Radiol 17(1 Pt A): 75-77

42. Waite S, Grigorian A, Alexander RG, Macknik SL, Carrasco M, Heeger DJ, Martinez-Conde S (2019) Analysis of perceptual expertise in radiology - current knowledge and a new perspective. Front Hum Neurosci 13:213

43. Bai HX, Hsieh B, Xiong Z, Halsey K, Choi JW, Tran TML, Pan I, Shi L-B, Wang D-C, Mei J et al: Performance of radiologists in differentiating COVID-19 from viral pneumonia on chest CT. Radiology, 0(0):200823

44. Wiggins G (2011) A true test: toward more authentic and equitable assessment. Phi Delta Kappan 92(7):81-93

45. iOS device compatibility reference. Retrieved July 10, 2020, from [https://developer.apple.com/library/archive/documentation/ DeviceInformation/Reference/iOSDeviceCompatibility/Displays/ Displays.html]

46. Compare - Official Samsung Galaxy Site. Retrieved July 10, 2020, from [https://www.samsung.com/global/galaxy/comparesmartphones/]

47. Toomey RJ, Rainford LA, Leong DL, Butler ML, Evanoff MG, Kavanagh EC, Ryan JT (2014) Is the iPad suitable for image display at American Board of Radiology examinations? AJR Am J Roentgenol 203(5):1028-1033

Publisher's note Springer Nature remains neutral with regard to jurisdictional claims in published maps and institutional affiliations. 